\title{
Exhaust emission and fuel consumption characteristics of light duty under different driving cycles
}

\author{
Peilin Geng ${ }^{*}$, Le Liu, Yuwei Wang, and Xionghui Zou \\ China Automotive Technology and Research Center Co., Ltd., Tianjin 300300
}

\begin{abstract}
Keywords: testing cycle, light vehicle, exhaust emission, fuel consumption.
\end{abstract}

\begin{abstract}
This paper focuses on light duty of china 6 with the same emission control technology. three vehicles, with different engine displacements, were selected to study the emission and fuel consumption characteristics under three test cycles of NEDC, WLTC and CLTC. The results show that the emissions of CO, THC and NOx under WLTC cycle are minimum, compared with the NEDC and CLTC circulation. with the decrease of the engine displacement, the difference of $\mathrm{CO}$ and THC emissions increases among different cycles, which shows small displacement engine vehicles are greatly affected by driving cycles. Compared with other testing conditions, the PN emissions are relatively larger, but the difference of PN emissions is very small among the three test cycles.The fuel consumption of the WLTC test cycle is the smallest among the three cycles. As the engine displacement decreases, the fuel consumption difference decreases, indicating that the fuel consumption of large displacement engine vehicles is greatly affected by the cycle condition.
\end{abstract}

\section{Introduction}

Vehicle exhaust emissions has increasingly become the main source of urban air pollution. Light vehicle is a major contributor to $\mathrm{CO}$ and $\mathrm{HC}$ emissions of vehicles and close to the crowd, which has a directly impact on human health. The vehicle fuel consumption and emissions are mainly carried out with the specific cycle conditions on the test room.

Vehicle emissions and fuel consumption are directly affected by test cycle conditions. At present, WLTC(World Light Vehicle Test Cycle) is used in China's light duty, replacing the previous NEDC (New European driving cycle). WLTC is a transient cycle and made up of four parts, namely low, medium, high and extra high, Which can better reflect the actual driving situation of the vehicle.However, the research shows that the light vehicles in China are mainly used in urban and suburban areas (the time proportion is about $76 \%$,

\footnotetext{
* Corresponding author: gengpeilin@catarc.ac.cn
} 
mileage proportion is about $56 \%)$. The average speed is low $(29 \mathrm{~km} / \mathrm{h})$, and the idle speed ratio is high (about $22 \%$ ), while the four data of WLTC are $57 \%, 43 \%, 46.5 \mathrm{~km} / \mathrm{h}$ and $13.2 \%$, which obviously deviates from the actual situation in China. In order to better reflect the emission of China light vehicles, it is urgent to explore a test cycle that can more represent china's driving cycle. Therefor, CLTC (China automobile testing cycle) was developed and makes up for the lack of its own test cycle in China for a long time,which was issued in National standard Announcement No. 13 of 2019. CLTC provides more choices for the measurement of emissions and fuel consumption of China, and also provides more practical test condition for the electric vehicle endurance measurement.

Scholars at home and abroad have never stopped exploring and studying the test cycle, most of which focus on the emission and fuel consumption characteristics of NEDC, WLTC andFTP-75. Li et al. found that the different effects of NEDC, WLTC and FTP-75 on emission are mainly reflected in cold start condition, transient condition and high speed condition[1]. Zhang et al.[2] further analyzed the differences between the transient pollutants and the weighted pollutants under the three test cycles of NEDC, WLTC and FTP-75, and concluded that due to the frequent changes of WLTC cycle and the high average speed, the emissions of CO and NOx are the highest, and due to the short driving distance of NEDC, the weighted HC emissions are the highest, and the transient pollutants of FTP-75 are the highest, but the final pollutant emission is not high after the weighted calculation of cold and hot stage. At present, CLTC cycle has been applied to all heavy-duty commercial vehicles and new energy vehicles, and will be gradually introduced into emission, noise or other fields. However, there are few reports on the adaptability of CLTC cycle in light duty in China.

In order to find out the similarities and differences between NEDC, WLTC and CLTC on the emission and fuel consumption of various pollutants, and provide technical support for the application of CLTC cycle in light vehicles in China, three vehicles of china 6 with the same emission control route are carried out. The experiment uses the same loading mode and respectively run three test cycles, and the transient emission, weighted emission and fuel consumption of various pollutants under the three cycles are compared and analyzed.so as to provide technical support for the application of CLTC cycle in light vehicles in China.

\section{Experimental setup and method}

\subsection{Test cycle}

In this test, three test cycles are selected to test the china 6 light duty vehicle. The test cycles are NEDC, WLTC and CLTC. The specific parameters are shown in Fig. 1. 


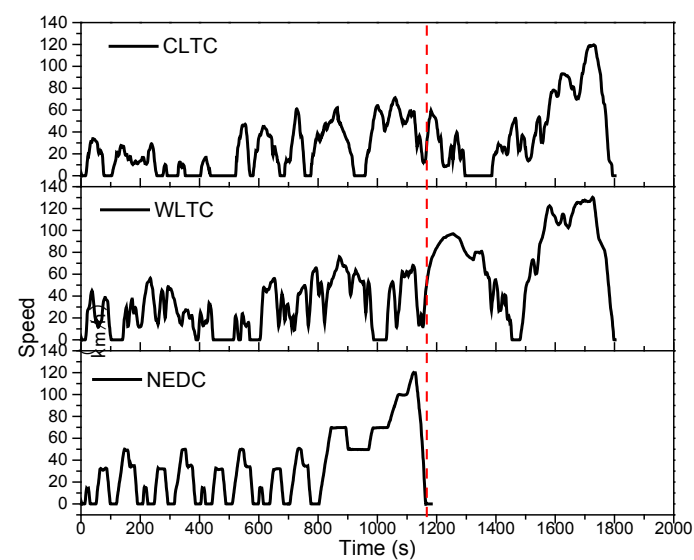

Fig. 1. Comparison diagram of three test cycle conditions for NEDC, WLTC and CLTC.

As shown in Fig.1, both WLTC and CLTC are transient and variable conditions, which are composed of different speed segments, representing the operation of vehicles at low speed, medium speed and high speed, while NEDC belongs to steady-state conditions, which is made up of urban and suburban speed segments. The running time of both WLTC and CLTC cycles was 1800 s, which was 620 s longer than the testing time of NEDC condition. In this three test cycles, the maximum speed and average speed of WLTC are the largest, followed by NEDC, and finally CLTC, and the maximum acceleration of WLTC is the largest, followed by CLTC, and finally NEDC. The specific parameters are shown in table 1 .

Table 1. Main parameters of NEDC, WLTC and CLTC test cycles.

\begin{tabular}{|c|c|c|c|c|c|c|}
\hline $\begin{array}{c}\text { Test } \\
\text { cycle }\end{array}$ & $\begin{array}{c}\text { Running } \\
\text { time }(\mathrm{s})\end{array}$ & Mileage $(\mathrm{km})$ & $\begin{array}{c}\text { Maximum } \\
\text { speed }(\mathrm{km} / \mathrm{h})\end{array}$ & $\begin{array}{c}\text { Average } \\
\text { speed }(\mathrm{km} / \mathrm{h})\end{array}$ & $\begin{array}{c}\text { Idle } \\
\text { time }(\%)\end{array}$ & $\begin{array}{c}\text { Maximum } \\
\text { acceleration(m/s2) }\end{array}$ \\
\hline WLTC & 1800 & 23.27 & 131.3 & 46.5 & 13.2 & 1.67 \\
\hline CLTC & 1800 & 14.48 & 114 & 29 & 22.1 & 1.47 \\
\hline NEDC & 1180 & 11.03 & 120 & 33.6 & 24.8 & 1.04 \\
\hline
\end{tabular}

\subsection{Test vehicle}

In the experiment, Three light-duty vehicles were selected, whose engine displacement was $1.5 \mathrm{~L}-2.0 \mathrm{~L}$, covering the mainstream engine displacement in the market. All three vehicles adopted the china 6 mainstream technology control route of turbocharged direct injection with GPF. The specific parameters of the test vehicles are shown in table 2.

Table 2. Parameters of test vehicles.

\begin{tabular}{cccc}
\hline Parameter & Vehicle 1 & Vehicle 2 & Vehicle 3 \\
\hline Engine type & Gasoline & Gasoline & Gasoline \\
Displacement/L & 2.0 & 1.8 & 1.5 \\
Intake type & Supercharge & Supercharge & Supercharge \\
Injection type & GDI & GDI & GDI \\
Gasoline particulate filter & Yes & Yes & Yes \\
\hline
\end{tabular}

\subsection{Experimental method}

The test is carried out on the rotating hub. The exhaust equipment used is HORIBA gas analyzer and AVL particle counter (PNC), which is used to analyze THC, CO, NMHC, 
NOx, PN and PM. The fuel consumption is obtained by reversing the carbon emission from the exhaust gas. In the experiment, all the vehicles were loaded in the same way. The three test cycles, NEDC, WLTC and CLTC, were used to test the pollutant emissions and instantaneous modal quantities in different cycle conditions. The schematic diagram of experimental test bench is shown in Fig. 2.

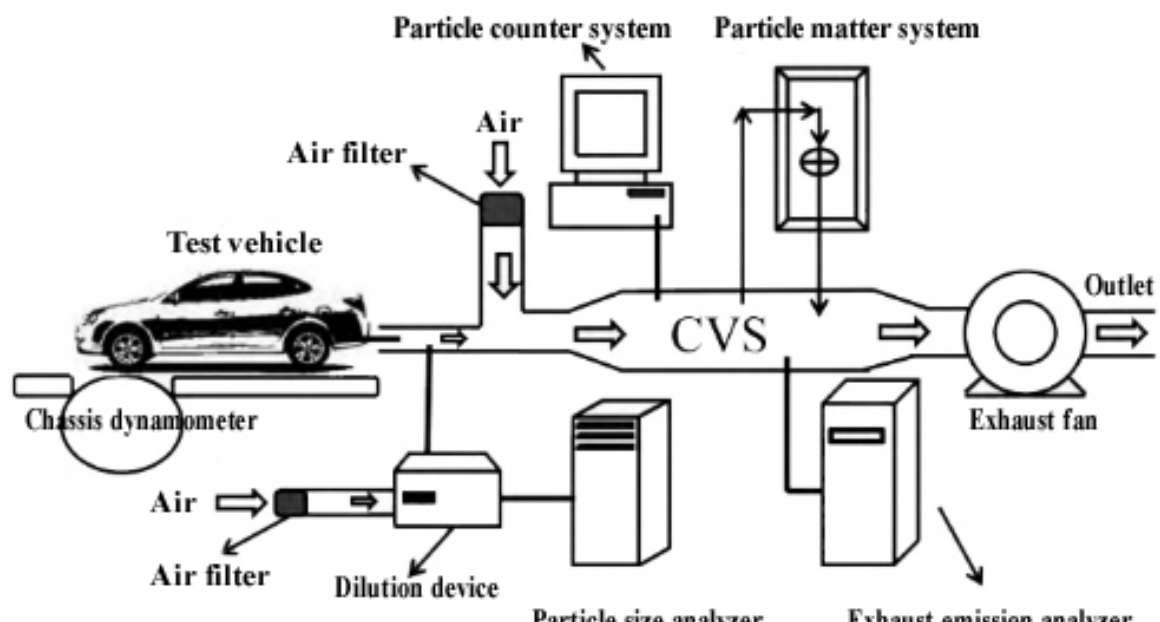

Fig. 2. The schematic diagram of experimental test bench.

\section{The influence of test cycle on emission characteristics}

\subsection{The influence of test cycle on instantaneous emission}

In order to analyze the influence of different cycle conditions on the instantaneous emission of exhaust pollutants, a turbocharged direct injection vehicle (vehicle 3) with GPF was selected. The test is carried out in the loading mode of china 6 under three test cycles, and the test results are shown in Fig. 3-5.

Under the three test cycle conditions, the emissions of $\mathrm{CO}, \mathrm{THC}$, NOx and PN increased sharply within 100s of cold start. In the subsequent test process, the emissions of $\mathrm{CO}$ and $\mathrm{THC}$ were in a stable and low emission state, while the emissions of NOx only produced a small amount of emissions in the acceleration stage. At other times, the emissions were close to zero, and the emissions of PN increased rapidly with the rapid acceleration of each speed. Therefore, in the four kinds of pollution emissions, the PN emissions are not only produced in a large amount in the cold start stage, but also have a great correlation with the acceleration of vehicles.

As can be seen from the graph, the four pollutants are mainly concentrated in the $100 \mathrm{~s}$ time after the engine starts. Taking CLTC test cycle as an example, in 100s of cold start time, $\mathrm{CO}$ emissions account for $65.2 \%$ of the total, THC emissions account for $23.9 \%$, NOx emissions account for $53.6 \%$ of the total, and PN emissions account for $19.8 \%$ of the total. It can be seen that in the cold start phase, which only accounts for $5.5 \%$ of the total test cycle, $\mathrm{CO}$ and NOx emissions contribute more than 50\%, THC and PN emissions contribute about $20 \%$, which is a significant contribution. The reason is that in the cold start stage, the temperature of the three-way catalyst is relatively low, which has not reached the ignition temperature of the three-way catalyst. In this stage, the catalyst work efficiency is relatively low. 


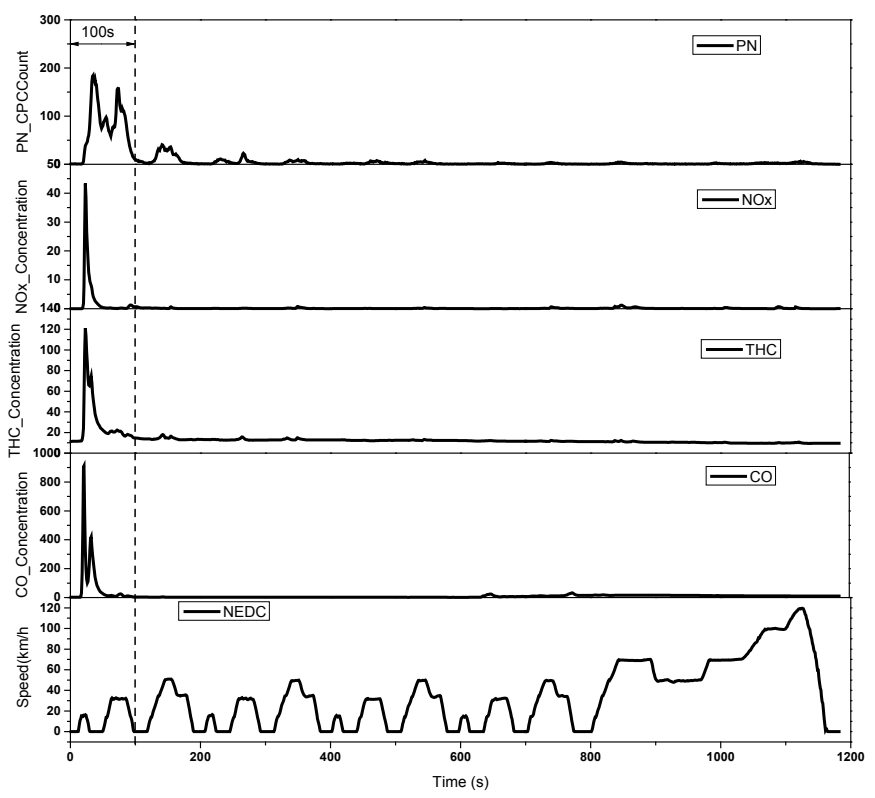

Fig. 2. Instantaneous emission of various pollutants under NEDC.

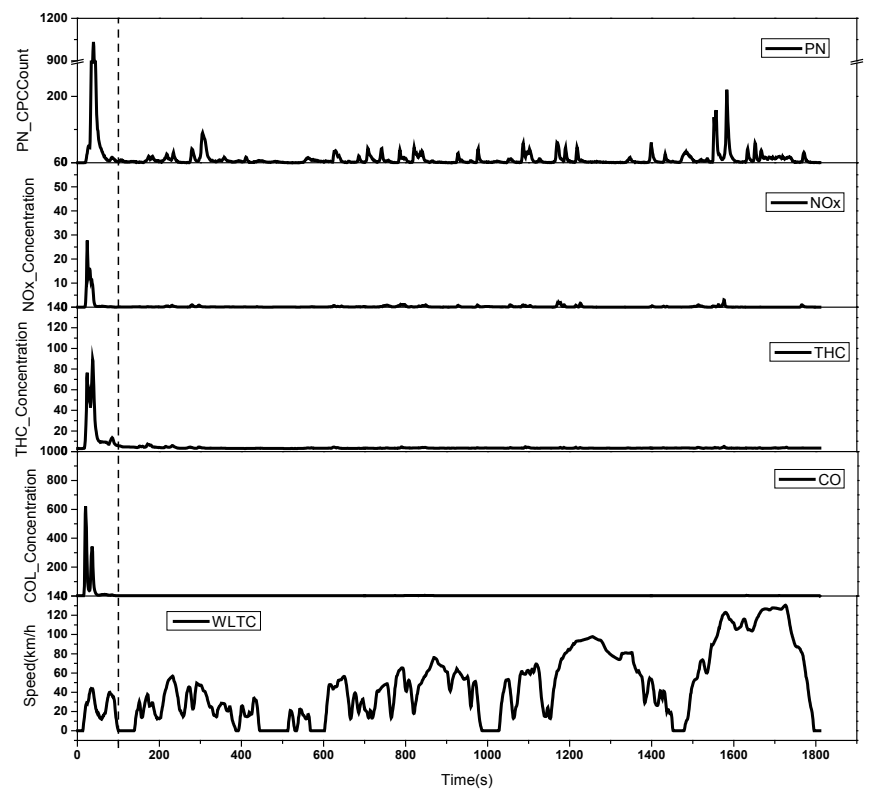

Fig. 4. Instantaneous emission of various pollutants under WLTC. 


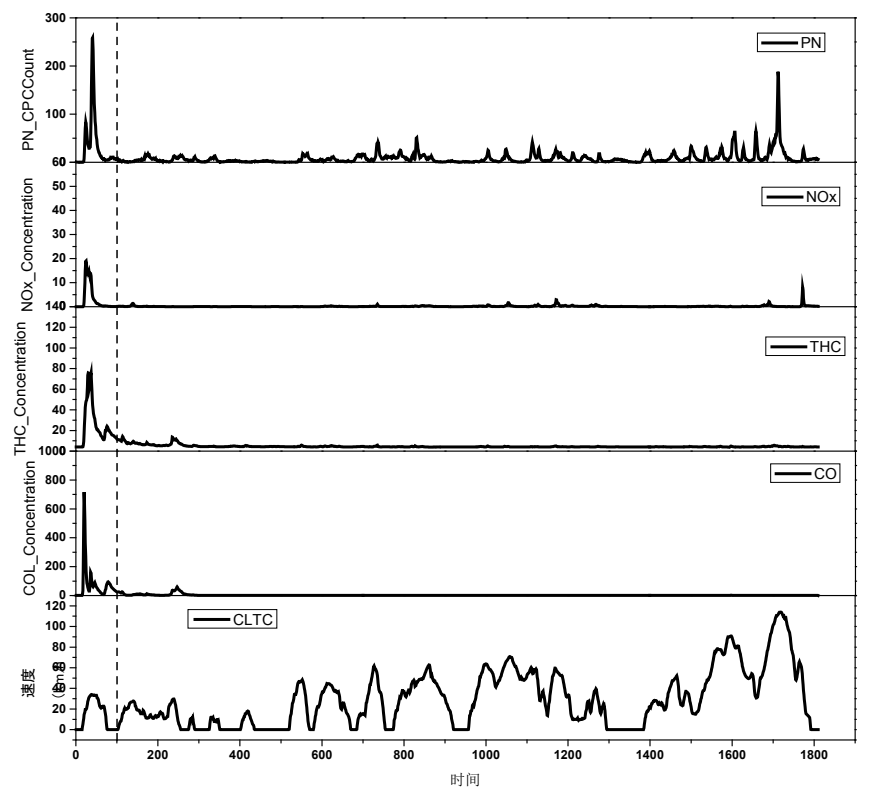

Fig. 5. Instantaneous emission of various pollutants under CLTC.

The cumulative instantaneous pollutant emissions under different cycle conditions are shown in Fig.5. The total transient pollutant emission is obtained by accumulating the transient data of the whole test cycle, and then dividing the driving mileage of the test cycle. As shown in the above figure, The CO, THC and NOx emissions in the WLTC are the least, and the emissions in the NEDC are the largest, while the emissions in the CLTC are between the NEDC and WLTC cycles. This is because the peak values of CO, THC and NOx emissions of the NEDC cycle in the cold start stage, which accounts for a large proportion of the total emissions under the three cycles, are the largest, and the cycle mileage is the smallest, so the cumulative instantaneous pollutant emissions of the NEDC cycle are the largest. The peak values of WLTC and CLTC emissions of the three gas pollutants are the same, but the driving mileage of CLTC cycle is less than WLTC, so the cumulative modal pollutant emission of CLTC cycle is higher than that of WLTC cycle. PN emissions are not only related to the cold start phase, but also to the transient acceleration. The acceleration time of NEDC is less, and it is mostly steady state, so the cumulative emissions of PN in NEDC cycle are the smallest compared with WLTC and CLTC cycle, while the cumulative emissions of PN in WLTC and CLTC are almost the same.

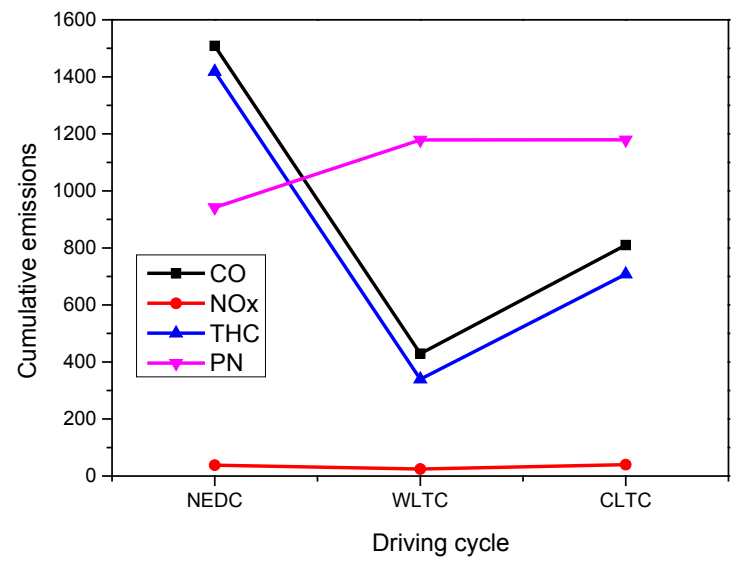


Fig. 6. Transient accumulation of pollutants under different cyclic conditions.

\subsection{The influence of test cycle on pollutant emission and fuel consumption}

In order to study the influence of three test cycles on pollutant emission and fuel consumption of national six light vehicles, three kinds of mainstream engine displacement vehicles were selected. The three vehicles is vehicle 1 , vehicle 2 and vehicle 3 , with engine displacement of $2.0 \mathrm{~L}, 1.8 \mathrm{~L}$ and $1.5 \mathrm{~L}$ respectively. At the same time, the three vehicles all adopt supercharging, direct injection and GPF technology.

\subsubsection{Emission characteristic}

The emission characteristics of THC, CO and NOx of the three vehicles under the NEDC, WLTC and CLTC are shown in Fig. 7-9. Except for the same NOx emission of vehicle 1 under the three test cycles, the THC, CO and NOx emissions of three vehicles have the same rule under the three test cycles. The emissions of WLTC cycle are the smallest, and the emissions of NEDC cycle and CLTC cycle are basically the same, Which is also consistent with the transient conclusion in Fig. 3-5. This is because the emissions of THC, $\mathrm{CO}$ and NOx are mainly affected by cold start, and the cold start of NEDC cycle accounts for a large proportion[4], at the same time, the mileage of NEDC is also short, which leads to the maximum emission of three pollutants under NEDC cycle. The driving mileage of WLTC cycle is the longest, and the instantaneous emission of pollutants in cold start phase is equivalent to that of CLTC, so the emissions of THC, CO and NOx measured by WLTC cycle are relatively low.

Among the three vehicles, the THC and CO emissions are lower than the china 6B limit values of light duty under all the three test cycles, but the NOx emission of vehicle 2 under CLTC cycle increases significantly, which is 4.3 times of china $6 \mathrm{~b}$ limit value and 5.3 times of WLTC cycle, indicating that NOx emission is more sensitive to the test cycle. It is also found that in addition to the NOx emission of vehicle 2, with the reduction of engine displacement, the difference of pollutant emissions under the three cycles is also greater, which shows that the cycle conditions have a greater impact on the pollutant emissions of small displacement vehicle.

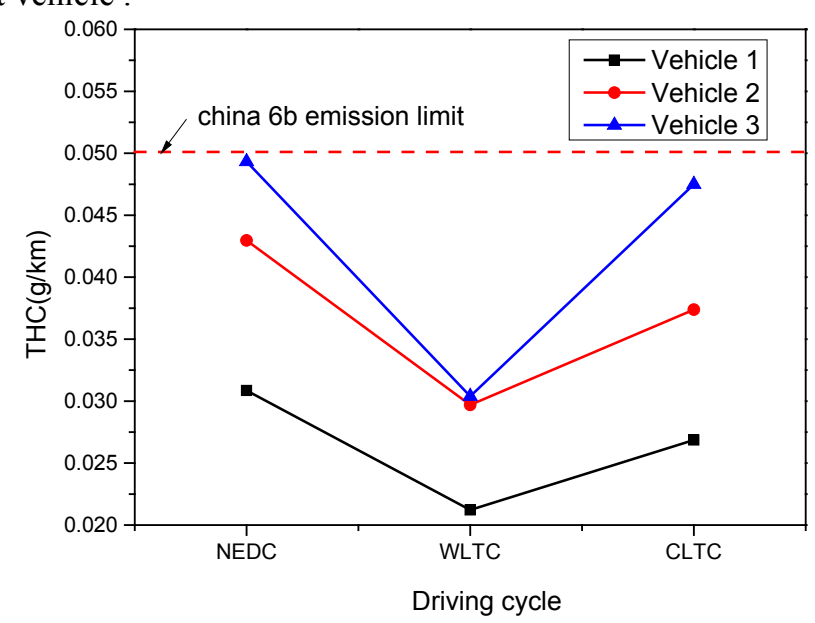

Fig. 7. THC emissions under different test cycles. 


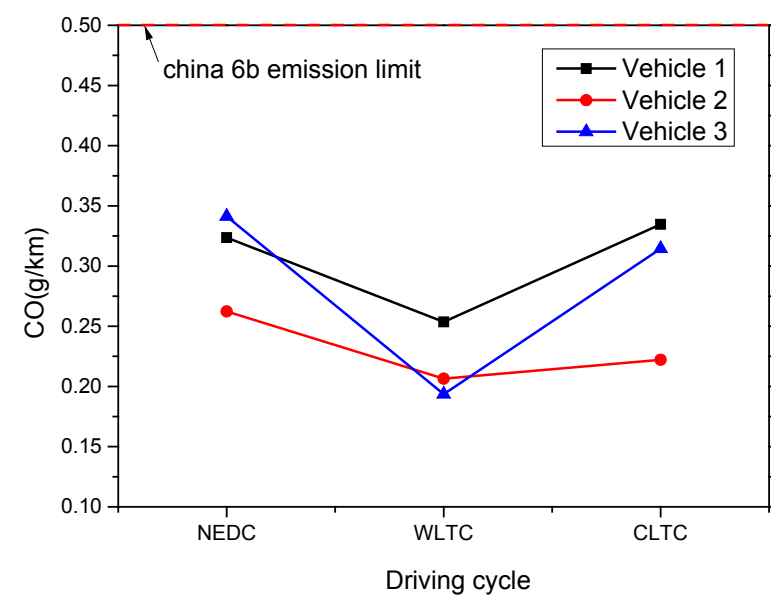

Fig. 8. CO emissions under different test cycles.

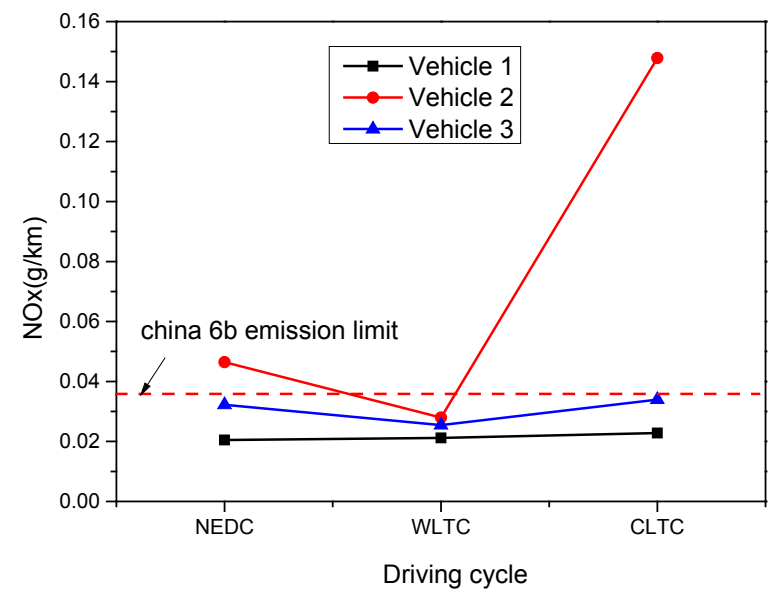

Fig. 9. NOx emissions under different test cycles.

Figure10 presents the PN emission characteristics of the three vehicles under the NEDC, WLTC and CLTC . As shown in Fig. 10, the PN emission rules of three test cycles is opposite to THC, CO and NOx. The PN emission of WLTC test cycle is the largest among the three test cycles. This is because PN is generated in large numbers in the cold start stage, and is also generated in every acceleration phase. Compared with NEDC and CLTC cycles, WLTC has the most frequent acceleration times and the largest acceleration, which leads to the generation of a large number of particles. It is also found that compared with the other two relatively small engine displacements, the $2.0 \mathrm{~L}$ displacement has the largest PN emissions, indicating that the large engine displacement maybe bring greater PN emissions. 


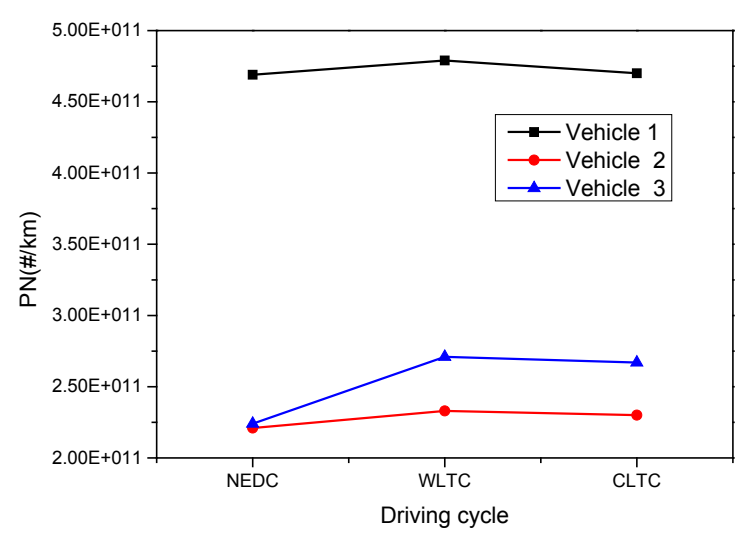

Fig. 10. PN emissions under different test cycles.

\subsubsection{Fuel consumption characteristic}

Figure11 presents the fuel consumption characteristics of the three vehicles under the NEDC, WLTC and CLTC. As shown in Fig. 10, the fuel consumption of the whole vehicle decreases gradually with the reduction of engine displacement, and the fuel consumption of the WLTC cycle is the minimum. This is because compared with the NEDC cycle, the start phase effect of WLTC is weakened, and the proportion of stopping time is shortened, which is closer to the economic driving speed. At the same time, the driving mileage of WLTC cycle is larger, which also makes the calculation result much smaller[6]. The driving mileage of CLTC is shorter than that of WLTC cycle, and the average speed is lower than that of WLTC cycle, all of which increase fuel consumption, so the fuel consumption of CLTC cycle is larger than that of WLTC cycle.

It is also found that the fuel consumption of vehicles with large displacement engine is greatly affected by the cycle conditions. For $2.0 \mathrm{~L}$ vehicles (Vehicle 1), the maximum and minimum fuel consumption difference between the three cycles is $2.12 \mathrm{~L}$, and for $1.8 \mathrm{~L}$ vehicles (Vehicle2), the maximum and minimum fuel consumption difference of the three cycles is $1.41 \mathrm{~L}$, and for $1.5 \mathrm{~L}$ vehicles (Vehicle 3 ), the maximum and minimum fuel consumption difference of the three cycles is $0.45 \mathrm{~L}$.

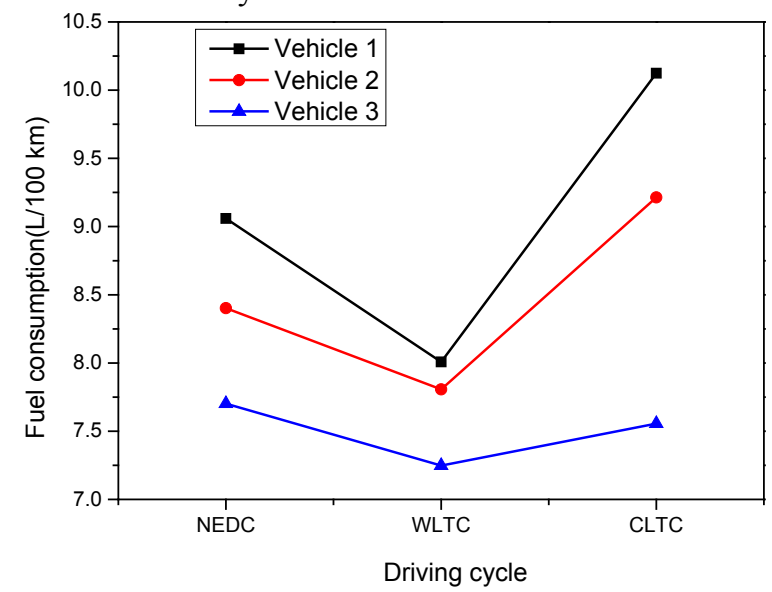

Fig. 11. Fuel consumption under different test cycles. 


\section{Conclusion}

Based on study of exhaust emission and fuel consumption characteristics of china 6 light duty under different test cycles, it can be concluded as follows:

1) Among the three test cycles of NEDC, WLTC and CLTC, the emissions of CO, THC and NOx are the smallest under WLTC cycle, while the PN emissions are relatively large.The emissions of $\mathrm{PN}$ in the three test cycles have little difference.

2) The emissions of CO and THC under the CLTC cycle are between NEDC and WLTC cycles, and NOx emission is the largest among the three test cycles.

3) Compared with the NEDC and CLTC cycles, the fuel consumption of the WLTC test cycle is the smallest. As the engine displacement decreases, the fuel consumption difference decreases among the three cycles, indicating that the fuel consumption of large displacement engine vehicles is greatly affected by the cycle conditions.

This work was financially supported by the Construction of Public Service Platform for Vehicle Emission/Energy Consumption Information System(Improvement of public service capacity of Industrial Technology Foundation 2018), the Integrated Technology of Particulate Matter Capture and Clean Emission for Gasoline Vehicles Projects (No.: 2017YFC0211004 and 2017YFC0211005).

\section{References}

1. Li Yanguang.Li Xue,Lei Chunqing. study on the impact of emission test cycle on light vehicle emissions [J]. China High Tech Entrprises,2016(9):86-87.

2. Zhang Pengyu, Zhang Xinyu, He Lijun, et al. Analysis of Pollutant Emission Characteristics for Light-duty Vehicles under Different Test Cycles[J]. Vehicle \& Power Technology, 2016.

3. Guo Qianli, Zhao Donchang, Chen Pin,et al. Comparison of WLTC \& NEDC and Preliminary Study of Their Impact on Automobile Fuel Consumption [J]. Chinese Journal of Automotive Engineering,2017(3):44-52.

4. Marotta A, Pavlovic J , Ciuffo B , et al. Gaseous Emissions from Light-Duty Vehicles: Moving from NEDC to the New WLTP Test Procedure[J]. Environmental Science \& Technology, 2015, 49(14):8315-8322.

5. Piotr B, Joseph W, Andrzej S. Szczotka. Exhaust Emissions of Gaseous and Solid Pollutants Measured over the NEDC, FTP-75 and WLTC Chassis Dynamometer Driving Cycles [C]. SAE Technical Paper. 2016-01-1008:

6. Bielaczyc P, Woodburn J, Szczotka A. A comparison of carbon dioxide exhaust emissions and fuel consumption for vehicles tested over the NEDC, FTP-75 and WLTC chassis dynamometer test cycles [C]. SAE Technical Paper. 2015-01-1065. 